\title{
Particles as Wilson lines of gravitational field
}

\author{
L. Freidel, J. Kowalski-Glikman` and A. Starodubtsev ${ }^{\ddagger}$
}

August 30, 2018

\begin{abstract}
Since the work of Mac-Dowell-Mansouri it is well known that gravity can be written as a gauge theory for the de Sitter group. In this paper we consider the coupling of this theory to the simplest gauge invariant observables that is, Wilson lines. The dynamics of these Wilson lines is shown to reproduce exactly the dynamics of relativistic particles coupled to gravity, the gauge charges carried by Wilson lines being the mass and spin of the particles. Insertion of Wilson lines breaks in a controlled manner the diffeomorphism symmetry of the theory and the gauge degree of freedom are transmuted to particles degree of freedom.
\end{abstract}

\footnotetext{
*Perimeter Institute for Theoretical Physics, Waterloo, Canada; Laboratoire de Physique, École Normale Supérieure de Lyon, Lyon , France; lfreidel@perimeterinstitute.ca

${ }^{\dagger}$ Institute for Theoretical Physics, University of Wroclaw, Poland; jurekk@ift.uni.wroc.pl

${ }^{\ddagger}$ Institute for Theoretical Physics, University of Utrecht, The Netherlands; A.Starodubtsev@phys.uu.nl
} 


\section{Introduction}

"Geometry tells matter how to move; matter tells geometry how to curve" - this simple sentence encompasses the main physical message of general theory of relativity. Yet, as any great idea, it contains the seed of problems: there is an explicit dichotomy in this statement - the division of the physical world into two entities, matter and geometry.

There is an old dream, preceding the idea of unification of all interactions, to unify geometry and matter, usually in the guise of geometrization of matter (though string theory seems to take the completely opposite stance, attempting to "matterize" gravity.) If gravitational field is geometry is it that not reasonable to expect that all other physical objects could be described in terms of geometry of some sort as well? In this paper we aim to give an affirmative answer to this question. To be more precise we show that point particles, with momentum and spin, can be described as Wilson lines of an appropriate connection.

The particle can be described by the momentum and spin it carries. These are charges of Poincaré group, which is also the gauge group of gravity with vanishing cosmological constant. However recent developments indicate that in order to construct a reasonable theory of (quantum) gravity one presumably should include an infrared regulator in the form of cosmological constant. Thus the minimal geometrical model must be based on the gauge group being de Sitter group, $\mathrm{SO}(4,1)$ in four dimensions. The vanishing (or small - as it is the case in our universe) cosmological constant setting can be obtained then by taking an appropriate limit of such a theory. Fortunately the charges of this group can be still interpreted as momentum and spin, so coupling of gravity understood as a gauge theory of $\mathrm{SO}(4,1)$ can be naturally coupled to matter. This also raised the possibility that matter could be understood in terms of some specific configurations of gravitational field. In this paper we show how this construction can be realized explicitly.

In the next section, following [1] we recall how gravity can be constructed as a constrained topological field theory. In section 3, using the formalism of Balachandran et. al. 2] we shortly review the way the point particles can be coupled to external gauge field. We show that in the case of the $\mathrm{SO}(4,1)$ gauge group the resulting equations reduce to the standard Mathisson-Papapetrou form, in the appropriate limits. In the following section we show that also the gravitational field equations acquire the correct form, with energy momentum tensor, and the source of torsion being point-like particles with appropriate energy-momentum and spin. Section 5 explains how particles could be understood in terms of Wilson lines. We 
conclude this paper with some comments, and with an appendix containing some useful formulas.

\section{The action of gravity and topological field the- ory}

In this section, following [1] we present for the reader's convenience the action for gravity as an action of a perturbed topological BF system. This formulation is an extension of the original work of Mac Dowell and Mansouri [3] who expressed gravity as a $\mathrm{SO}(4,1)$ gauge theory. We focus on $\mathrm{SO}(4,1)$ since it concerns Lorentzian gravity with a positive cosmological constant which is clearly the most physically interesting case. Everything we say in this paper can be readily translated to the case of negative cosmological constant or Euclidean gravity. Note however that we definitely need a non zero cosmological constant for this formalism to make sense.

The gravitational field is encoded it in a $\mathrm{SO}(4,1)$ (de Sitter) connection

$$
\mathrm{A}_{\mu}=\left(\frac{1}{2 l} e_{\mu}{ }^{a} \gamma_{a} \gamma+\frac{1}{4} \omega_{\mu}^{a b} \gamma_{a b}\right)
$$

where the index $a$ runs from 0 to 3 . In this formula, gamma matrices $\gamma_{a} \gamma / 4$, $\gamma_{a b} / 4$ form a representation of generators $T^{I J}\left(I, J=0, \ldots 4, \gamma=\gamma_{4}\right)$ of the Lie algebra so $(4,1) . \quad e_{\mu}{ }^{a}$ is the frame field from which the metric is constructed and $\omega_{\mu}{ }^{a b}$ is the spin connection. When written in terms of the so $(4,1)$ generators the connection reads $\mathrm{A}=A^{I J} T_{I J}$. Connection has a mass dimension 1 whereas the frame field is dimensionless; this is the reason why a length scale $l$ appears in the expression of the components of A representing the frame field. As we will see in order to recover the usual gravitational dynamics this length scale has to be the cosmological length $l$ related to the cosmological constant by

$$
\frac{1}{l^{2}}=\frac{\Lambda}{3}
$$

To formulate the theory we also need a two form valued in the Lie algebra so $(4,1)$ denoted by $\mathrm{B}=B_{\mu \nu}^{I J} T_{I J} \mathrm{~d} x^{\mu} \wedge \mathrm{d} x^{\nu}$. In terms of this two form and connection $A$ the action takes the form

$$
-\frac{1}{2} S=\int \operatorname{Tr}\left(\mathrm{B} \wedge \mathrm{F}(\mathrm{A})-\frac{i \alpha}{2} \mathrm{~B} \wedge \mathrm{B} \gamma-\frac{\beta}{2} \mathrm{~B} \wedge \mathrm{B}\right)
$$

which can be rewritten in components as

$$
S=\int\left(B_{I J} \wedge F^{I J}-\frac{\alpha}{4} B_{I J} \wedge B_{K L} \epsilon^{I J K L 4}-\frac{\beta}{2} B^{I J} \wedge B_{I J}\right)
$$


This action can be shown [1] to be equivalent to the action of General Relativity, for $\alpha \neq 0$.

In order to solve the equation of motion for $B$ it is convenient to introduce the operators

$$
\mathcal{P}_{ \pm}{ }^{i j}{ }_{k l}=\left[ \pm \frac{\alpha}{2} \epsilon^{i j}{ }_{k l}+\beta \delta_{k l}^{i j}\right], \quad \mathcal{P}_{-}{ }^{i j}{ }_{k l} \mathcal{P}_{+}{ }^{k l}{ }_{m n}=\left(\alpha^{2}+\beta^{2}\right) \delta_{m n}^{i j}
$$

The field equations for B following from (2.3) read

$$
\begin{aligned}
B^{i j} & =\frac{1}{\alpha^{2}+\beta^{2}} \mathcal{P}_{-}{ }^{i j}{ }_{k l} F^{k l}, \\
B^{4 i} & =\frac{1}{\beta} F^{4 i} .
\end{aligned}
$$

These equations are algebraic, so we can substitute them back to (2.3) to obtain

$$
S=\int\left(\frac{-\alpha}{4\left(\alpha^{2}+\beta^{2}\right)} F^{i j} \wedge F^{k l} \epsilon_{i j k l}+\frac{\beta}{2\left(\alpha^{2}+\beta^{2}\right)} F^{i j} \wedge F_{i j}+\frac{1}{\beta} F^{5 i} \wedge F_{5 i}\right)
$$

The curvatures of connection A are decomposed as follows

$$
\begin{array}{r}
F^{i j}(\mathrm{~A})=R^{i j}(\omega)-\frac{1}{l^{2}} e^{i} \wedge e^{j} \\
F^{i 4}(\mathrm{~A})=\frac{1}{l} d_{\omega} e^{i} .
\end{array}
$$

Using (2.9) and introducing the Nieh-Yan class

$$
C=d_{\omega} e^{i} \wedge d_{\omega} e_{i}-R^{i j} \wedge e_{i} \wedge e_{j}
$$

we can rewrite this action in terms of gravity variables

$$
\begin{gathered}
S=\widetilde{S}_{P}-\frac{\alpha}{4\left(\alpha^{2}+\beta^{2}\right)} \int R^{i j}(\omega) \wedge R^{k l}(\omega) \epsilon_{i j k l} \\
+\frac{\beta}{2\left(\alpha^{2}+\beta^{2}\right)} \int R^{i j}(\omega) \wedge R_{i j}(\omega)+\frac{1}{\beta} \int C .
\end{gathered}
$$

The last three terms are the integrals of Euler, Pontryagin, and Nieh-Yan classes. These are integer valued topological invariants with trivial local variation. The first term of action (2.10)

$$
\widetilde{S}_{P}=\frac{1}{2 G} \int R^{i j}(\omega) \wedge e^{k} \wedge e^{l} \epsilon_{i j k l}
$$




$$
-\frac{\Lambda}{12 G} \int e^{i} \wedge e^{j} \wedge e^{k} \wedge e^{l} \epsilon_{i j k l}+\frac{1}{G \gamma} \int R^{i j}(\omega) \wedge e_{i} \wedge e_{j}
$$

is the action of General Relativity with nonzero cosmological constant and a nonzero, dimensionless Immirzi parameter $\gamma$. The initial parameters $\alpha, \beta, l$ are related to the physical ones as follows

$$
\frac{1}{l^{2}}=\frac{\Lambda}{3}, \quad \alpha=\frac{G \Lambda}{3} \frac{1}{\left(1+\gamma^{2}\right)}, \quad \beta=\frac{G \Lambda}{3} \frac{\gamma}{\left(1+\gamma^{2}\right)} .
$$

Even if the term proportional to $\gamma^{-1}$ in (2.11) is not topological (its variation is non zero), it doesn't affect the classical equation of motion when $\gamma^{2} \neq-1$ unless the theory is coupled to fermions [4. We recover the usual metric gravity in the case $\gamma=0$, in this case the torsion is forced to vanish. The other extreme $\gamma=\infty$ correspond to Cartan-Weyl formulation of gravity [1]. It is important to note that in both cases we have $\beta=0$.

We see therefore that for $\alpha \neq 0$ the action (2.3) reproduces the action of General Relativity accompanied with a number of topological terms. This action makes it possible also to consider limits $\alpha \rightarrow 0$, in which equations of motion of General Relativity turn into those of topological field theory. Let us first consider $\alpha=0, \beta=0$ limit of (2.3) which, as it follows from (2.12), corresponds to the limit $G \rightarrow 0$. In this case we have to do with a pure $B F$ theory described by the action

$$
-\frac{1}{2} S_{0}=\int \operatorname{Tr} \mathrm{B} \wedge \mathrm{F}(\mathrm{A})
$$

whose equations of motion simply state that geometry is de Sitter flat

$$
F(A)=0
$$

- The topological theory (2.13) is invariant under two gauge symmetries, the standard

$$
\mathrm{A} \mapsto \mathrm{g}^{-1} \mathrm{Ag}+\mathrm{g}^{-1} \mathrm{dg}, \quad \mathrm{B} \mapsto \mathrm{g}^{-1} \mathrm{Bg}
$$

along with

$$
\mathrm{A} \mapsto \mathrm{A}, \quad \mathrm{B} \mapsto \mathrm{B}+d_{\mathrm{A}} \Phi
$$

where $d_{\mathrm{A}}=d+[\mathrm{A}, \cdot]$ is the covariant exterior derivative and $\Phi$ being an so $(4,1)$ valued one form.

Another possible limit is $\alpha=0, \beta \neq 0$, which involves large Immirzi parameter

$$
-\frac{1}{2} S_{0}=\int \operatorname{Tr}\left(\mathrm{B} \wedge \mathrm{F}(\mathrm{A})-\frac{\beta}{2} \mathrm{~B} \wedge \mathrm{B}\right)
$$


This theory is also invariant under (2.15), while (2.16) is replaced with

$$
\mathrm{A} \mapsto \mathrm{A}+\beta \Phi, \quad \mathrm{B} \mapsto \mathrm{B}+d_{\mathrm{A}} \Phi+\frac{\beta}{2}[\Phi, \Phi],
$$

which implies that spacetime geometry is arbitrary in the bulk.

In what follows we will consider mainly the most general case $\alpha, \beta \neq 0$, corresponding to the full dynamical gravity theory (with torsion and Immirzi parameter.) In some instances we will also discuss particular topological limits of this theory.

\section{Particle action and equations of motion}

In the formulation of the previous section gravity is formulated as a $\mathrm{SO}(4,1)$ gauge theory, of which only the $\mathrm{SO}(3,1)$ part is unbroken by the gravitational term proportional to $\alpha$. As shown in [5, 1, in the case of pure gravity, the formalism can be extended to be $\mathrm{SO}(4,1)$ gauge invariant with spontaneous symmetry breaking down to $\mathrm{SO}(3,1)$ Lorentz gauge invariance. The first goal of this paper is to show that matter can arise in the most natural way in this formalism by introducing the simplest possible term breaking the gauge symmetry of the theory in a localized way. The gauge degrees of freedom are then promoted to dynamical degree of freedom, and as we will show, reproduce the dynamics of a relativistic particle coupled to gravity. This realizes explicitly in four dimension the idea that matter (relativistic particles) can arise as a charged (under $\mathrm{SO}(4,1)$ ) topological gravitational defect. This strategy, well known in three dimensions, gives a new perspective where matter and gravity are geometrically unified [6] and was the key ingredient in the recent construction of the effective action of matter fields coupled to quantum three dimensional gravity [7].

An equivalent way to present the inclusion of matter in our context is to realize that the only natural way to couple a gauge field to localized excitation is by insertions of Wilson lines. Remarkably, the dynamics of these Wilson lines is the one of a relativistic particles. The formalism that allows to reach this conclusion was first developed by Balanchandran et. al. 2]. In this section we consider spinning particle moving in an external gravitational field, the full description of the particle(s) - gravity system will be described in the next section, while we return to Wilson lines in Section 5 .

The simplest possible localized gauge breaking coupling to the gravitational field ${ }^{1} \mathrm{~A}$ is obtained by choosing a worldline $P$ and a fixed element $K$

\footnotetext{
${ }^{1}$ We restrict in this paper to the gravitational coupling which has a clear physical
} 
of the so $(4,1)$ Lie algebra so as to have

$$
S_{P}(\mathrm{~A})=-\int \mathrm{d} \tau \operatorname{Tr}\left(\mathrm{KA}_{\tau}(\tau)\right)
$$

where $\tau$ parameterizes the world line $z^{\mu}(\tau)$ and $\mathrm{A}_{\tau}(\tau) \equiv \mathrm{A}_{\mu}(z(\tau)) \dot{z}^{\mu}$.

This action breaks gauge invariance and diffeomorphism symmetry ${ }^{2}$ In order to restore the symmetry at the particle location one promotes the gauge degree of freedom to the dynamical ones, which can be interpreted as Lorentz frame and particle position. This is similar to what happens in three dimension.

The 4-dimensional de Sitter group acts by conjugation on its Lie algebra, the orbits of this action being labelled by two numbers $(m, s)$ which are the mass and spin of the particle. For each orbit we choose a fixed representative element of the 4-dimensional de Sitter Lie algebra (for conventions see the Appendix)

$$
\mathrm{K} \equiv m l \gamma^{0} \gamma / 2+s \gamma^{2} \gamma^{3} / 4
$$

with obvious generalization in the case of massless particles.

The Lorentz Lie algebra so $(3,1)$ is identified with the subalgebra of so $(4,1)$ generated by $\gamma_{a b}$. The Lagrangian of a single particle propagating in a gravitational field is characterized by an embedding of its worldline $z(\tau)$ and a function $\mathrm{h}(\tau)$ valued in the Lorentz subgroup $\mathrm{h}=\exp \left(\alpha^{a b} \gamma_{a b} / 4\right)$. This function represents a Lorentz transformation from the rest frame, in which the Poincaré charges of the particle are described by the algebra element (3.2) to an actual frame, in which the particle has momentum $p$ and spin $s$ (see eq. (3.6) below.)

Let us denote by $A^{h}=h^{-1} A h+h^{-1} d h$ the corresponding gauge transformation of $A$. Then the lagrangian takes the simple form

$$
L(z, \mathrm{~h} ; \mathrm{A})=-\operatorname{Tr}\left(\mathrm{KA}_{\tau}^{\mathrm{h}}(\tau)\right) \quad S=\int d \tau L(z, \mathrm{~h} ; \mathrm{A}) .
$$

This lagrangian can be rewritten also in the following form

$$
L(z, \mathrm{~h} ; \mathrm{A})=-\operatorname{Tr}\left(\mathrm{JA}_{\tau}\right)+L_{1}(z, \mathrm{~h})
$$

interpretation. The coupling of string like sources to the $B$ field in $B F$ theory as been recently considered in [8, but its physical interpretation in the full theory is far from clarified.

${ }^{2}$ This is true for diffeomorphism that modify the worldline location. The action is still invariant under the residual symmetry consisting of diffeomorphisms that acts along the worldline, i-e reparametrisation invariance. 
where in the first term $\mathrm{J}$ is given by

$$
\mathrm{J} \equiv \mathrm{hKh}^{-1} \text {. }
$$

The components of $\mathrm{J}$ can be expressed in terms of the particle's momenta and spin

$$
\mathrm{J}=\frac{l}{2} p_{a} \gamma^{a} \gamma+\frac{1}{4} s_{a b} \gamma^{a b}
$$

The first term in equation (3.4) describes the covariant coupling between the particle and the A connection of the (constrained) BF theory, while the second

$$
L_{1}(z, \mathrm{~h})=-\operatorname{Tr}\left(\mathrm{h}^{-1} \dot{\mathrm{h}} \mathrm{K}\right),
$$

describes the dynamics of the particle.

This action is analogous to the spin part of the action for particle in three dimensional gravity $[\underline{6}$. The difference is that the gauge group is now SO $(4,1)$ which has two Casimir operators, mass and spin, and the information about the two Casimir operators is encoded in the extrinsic source $\mathrm{K}$, (3.2).

To put (3.4) into more conventional form let us rewrite it explicitly distinguishing the rotation transformations generated by $\gamma_{a b}, a, b=0, \ldots, 3$ and 'translation' transformations generated by $\gamma_{a} \gamma$. By introducing the scalars

$$
J^{I J}=-\operatorname{Tr}\left(\mathrm{J} \gamma^{I J} / 2\right), \quad \mathrm{J}=J^{I J} \gamma_{I J} / 4
$$

recalling that $A^{a 4}=\sqrt{\Lambda / 3} e^{a}$ and introducing momentum $p^{a}=\sqrt{\Lambda / 3} J^{a 4}$, and $\operatorname{spin} s^{a b}=J^{a b}$ we can rewrite (3.4) as

$$
L(z, \mathrm{~h} ; \mathrm{A})=\frac{1}{2}\left(A_{\tau}^{I J} J_{I J}\right)+L_{1}(z, \mathrm{~h})=e_{\tau}^{a} p_{a}+\frac{1}{2} \omega_{\tau}^{a b} s_{a b}+L_{1}(z, \mathrm{~h})
$$

Since $\mathrm{J}$ in the above equations is an element of the so( $(4,1)$ algebra, $J^{I J}$ must satisfy the constraints

$$
\frac{1}{2} J^{I J} J_{I J}=C_{2}
$$

and

$$
\frac{1}{16} J^{I J} J^{K L} \epsilon_{I J K L M} \epsilon^{M A B C D} J_{A B} J_{C D}=C_{4},
$$

where $C_{2}$ and $C_{4}$ are eigenvalues of quadratic and quartic Casimir operators of so $(4,1)$ algebra.

To see what is the physical meaning of $C_{2}$ and $C_{4}$ let us rewrite the equations (3.9) and (3.10) using the notations as in (3.8). Assuming that 
the cosmological constant $\Lambda$ is small and considering the leading order in $\Lambda$ we get

$$
C_{2}=\frac{1}{2} J^{a b} J_{a b}+J^{4 a} J_{4 a}=\frac{1}{2} s^{a b} s_{a b}+\frac{3}{\Lambda} p^{a} p_{a} \approx \frac{3}{\Lambda} p^{a} p_{a}
$$

and

$$
\begin{aligned}
C_{4} & =\left(\frac{1}{4} J^{a b} J^{c d} \epsilon_{a b c d}\right)^{2}+J^{a b} J^{4 c} \epsilon_{a b c d} \epsilon^{d e f g} J_{4 e} J_{f g} \\
& =\left(\frac{1}{4} s^{a b} s^{c d} \epsilon_{a b c d}\right)^{2}+\frac{3}{\Lambda} s^{a b} p^{c} \epsilon_{a b c d} \epsilon^{d e f g} p_{e} s_{f g} \\
& \approx \frac{3}{\Lambda} s^{a b} p^{c} \epsilon_{a b c d} \epsilon^{d e f g} p_{e} s_{f g},
\end{aligned}
$$

which is proportional to the length of the Pauli-Lubanski vector.

From the way the particles are coupled to the connection in (3.8) it is clear that $p_{\mu} \equiv e_{\mu}{ }^{a} p_{a}$ in the above equations is space-time momentum. From (3.11) one can see that the Casimir $C_{2}$ gives rise to the mass of the particle

$$
m^{2}=\frac{\Lambda}{3} C_{2} .
$$

The last equation for the mass relates two well-known problems in particle physics. Since the representation theory is labelled by integers, there is a natural unit and the most natural choice is to take the representation with minimal $C_{2}$. Under this assumption, explaining why the cosmological constant is small would also help to explain why masses of elementary particles are small, and vice versa.

In the center of mass frame, $p_{a}=(m, 0,0,0)$, the Casimir $C_{4}$ in (3.12) can be rewritten as

$$
C_{4}=\frac{3 m^{2}}{\Lambda} s^{i j} \epsilon_{i j k} \epsilon^{k l m} s_{l m}=C_{2} s^{i} s_{i}
$$

where $i, j, \ldots=1,2,3$ are $\mathrm{SO}(3)$-indices and $s^{i}=\epsilon^{i j k} s_{j k}$ is the spin in the rest frame of the particle. Thus we have the expression for the spin

$$
s^{2}=\frac{C_{4}}{C_{2}} .
$$

Consider now the equations of motion that follow from the Lagrangian (3.3). The variation over $h$ gives (ignoring total derivatives)

$$
\begin{aligned}
\delta L & =-\operatorname{Tr}\left(\mathrm{h}^{-1} \delta \mathrm{h}\left(\left[\mathrm{K}, \mathrm{A}^{\mathrm{h}}\right]\right)\right), \\
& =-\operatorname{Tr}\left(\delta \mathrm{hh}^{-1}\left(D_{\tau} \mathrm{J}\right)\right),
\end{aligned}
$$


where we introduced the $\mathrm{SO}(4,1)$ covariant derivative along the world-line of the particle

$$
D_{\tau} \equiv \frac{d}{d \tau}+\left[\mathrm{A}_{\tau}, \cdot\right]
$$

and $\mathrm{J}$ is defined by equation 3.5

Since $\mathrm{h}$ is restricted to be in the Lorentz subgroup $\mathrm{SO}(3,1)$ of $\mathrm{SO}(4,1)$ equations (3.16) constrain only the spin part of $\mathrm{J}$ and give the spin precession equation

$$
D_{\tau} J^{a b}=\nabla_{\tau} s^{a b}+e_{\tau}^{a} p^{b}-e_{\tau}^{b} p^{a}=0,
$$

with $\nabla_{\tau} \equiv \frac{d}{d \tau}+\left[\omega_{\tau}, \cdot\right]$ the Lorentz connection and $e_{\tau}{ }^{a}=e_{\mu}{ }^{a} \dot{z}^{\mu}$. Note that by construction the momenta and spin satisfy the orthogonality condition

$$
s^{a b} p_{b}=0
$$

In what follows we will also need the translational part of the current derivative

$$
\frac{1}{l} D_{\tau} J_{a}=\nabla_{\tau} p_{a}-\frac{1}{l^{2}} s_{a b} e_{\tau}^{b}
$$

The variation over $z$ gives

$$
\begin{aligned}
\frac{\delta L}{\delta z^{\mu}} & =\frac{d}{d \tau} \operatorname{Tr}\left(\mathrm{JA}_{\mu}\right)-\operatorname{Tr}\left(\mathrm{J} \partial_{\mu} \mathrm{A}_{\nu}\right) \dot{z}^{\nu} \\
& =\operatorname{Tr}\left(D_{\tau} \mathrm{JA}_{\mu}\right)-\operatorname{Tr}\left(\mathrm{J} F_{\mu \nu}(\mathrm{A})\right) \dot{z}^{\nu}=0
\end{aligned}
$$

where

$$
\begin{aligned}
& F_{\mu \nu}(\mathrm{A}) \equiv \partial_{\mu} \mathrm{A}_{\nu}-\partial_{\nu} \mathrm{A}_{\mu}+\left[\mathrm{A}_{\mu}, \mathrm{A}_{\nu}\right] \\
= & T_{\mu \nu}^{a} \gamma_{a} \gamma / 2+\left(R_{\mu \nu}^{a b}(\omega)-\frac{1}{l^{2}}\left(e_{\mu}{ }^{a} e_{\nu}^{b}-e_{\nu}{ }^{a} e_{\mu}{ }^{b}\right)\right) \gamma_{a b} / 4
\end{aligned}
$$

Here

$$
T^{a}=d e^{a}+\omega^{a}{ }_{b} \wedge e^{b}=T_{\mu \nu}{ }^{a} d x^{\mu} \wedge d x^{\nu} / 2
$$

is the torsion, while

$$
R^{a b}=d \omega+[\omega, \omega] / 2=R_{\mu \nu}^{a b} d x^{\mu} \wedge d x^{\nu} / 2
$$

is the Lorentz curvature.

If one uses equation (3.19), equations (3.22) written in components reads

$$
\left(\nabla_{\tau} p_{a}\right) e_{\mu}^{a}=\frac{1}{2} s_{a b} R_{\mu \nu}^{a b} \dot{z}^{\nu}+p_{a} T_{\mu \nu}^{a} \dot{z}^{\nu} .
$$


This is Mathisson-Papapetrou 9] equations describing the dynamic of spinning particle in the presence of torsion in an arbitrary gravitational background. When torsion is zero we recover the usual Mathisson-Papapetrou equation, when spin is also zero we recover the usual geodesic equation.

This equation can be written in the more usual form if one introduces the affine connection $\Gamma_{\mu \nu}{ }^{\rho}$, which is related to the spin connection $\omega_{\mu}^{a b}$ by the identity $\partial_{\mu} e_{\nu}^{a}+\omega_{\mu}^{a} b e_{\nu}^{b}=\Gamma_{\nu \mu}{ }^{\rho} e_{\rho}^{a}$. It can be written in terms of the Christofell symbol $\widehat{\Gamma}$ as $\Gamma_{\mu \nu \rho}=\widehat{\Gamma}_{\mu \nu \rho}+T_{\rho\{\mu \nu\}}-\frac{1}{2} T_{\mu \nu \rho}$ where $T_{\mu \nu \rho}=T_{\mu \nu}{ }^{a} e_{\rho a}$ is the torsion tensor, and the Mathisson-Papapetrou equation reads

$$
\nabla_{\tau} p_{\mu}=\frac{1}{2} s_{a b} R_{\mu \nu}^{a b} \dot{z}^{\nu},
$$

where $p_{\mu} \equiv p_{a} e_{\mu}^{a}$ and $\nabla_{\mu} p_{\nu} \equiv \partial_{\mu} p_{\nu}-\Gamma_{\mu \nu}{ }^{\rho} p_{\rho}$.

We see therefore that the $\mathrm{SO}(3,1)$ gauge transformation and the diffeomorphism symmetry is restored at the particle location while the gauge parameters acquire physical meaning being the Lorentz frame and particle position.

Above we restricted ourselves to $\mathrm{h}$ being valued in the Lorentz subgroup $\mathrm{SO}(3,1)$ of the full de Sitter group $\mathrm{SO}(4,1)$. We know however that if one further restrict ourselves to the topological case where $\alpha=0$, the bulk action is also invariant under de Sitter gauge transformations. In this case we can have a de Sitter covariant formulation of particle dynamics similar to that in $2+1$ gravity, where $\mathrm{h}$ has to be taken an element of $\mathrm{SO}(4,1)$. Let us try therefore to take $\mathrm{h} \in \mathrm{SO}(4,1)$, and see which additional equation will result. In doing so we would get from (3.16) an additional equation

$$
\nabla_{\tau} p_{a}=\frac{1}{l^{2}} s_{a b} e_{\tau}^{b}
$$

This equation is equivalent to eq. (3.22) provided that the following identity is satisfied along the particle worldline

$$
\frac{1}{l^{2}} s_{a b} e_{\mu}^{a} e_{\tau}^{b}=\frac{1}{2} s_{a b} R_{\mu \nu}^{a b} \dot{z}^{\nu}+\mathrm{p}_{a} T_{\mu \nu}{ }^{a} \dot{z}^{\nu} .
$$

This identity is satisfied for arbitrary particle if

$$
R_{\mu \nu}^{a b}-\frac{1}{l^{2}} e_{\mu}^{a} e_{\tau}^{b}=0, \quad T_{\mu \nu}^{a}=0
$$

i.e. if the background spacetime geometry is de Sitter. Such geometry holds in the limit in which $\alpha=0$, eq. (2.13). In this case the path integral quantization of the particle can be easily evaluated, see Section 5 . 
If one now consider the full gravity case where $\alpha \neq 0$, the bulk action also breaks the de Sitter invariance down to $\mathrm{SO}(3,1)$. As we will see in the next section, if one take into account the variation coming from the term proportional to $\alpha$ ( see eq. 4.6 and comments following it) the equation of motion obtained by making an $\mathrm{SO}(4,1)$ gauge transformation in the full gravity theory are consistent with the Mathisson-Papapetrou equations we derived above. Thus, analogously to what is shown in the context of pure gravity in [1] we expect a formulation of gravity coupled to particles where the the full de Sitter invariance is manifest, but spontaneously broken down to $S O(3,1)$ by classical solutions.

\section{Equations of motion for gravity - particle sys- tem}

Let us now consider the gravitational equation of motion when the gravitational field is coupled to a particle carrying Poincaré charge $\mathrm{J}$ (3.6). The action of this system will be given by the sum of actions (3.4) and (2.3). The equations of motion resulting from this action are as follows.

\section{$B$ equations}

$$
\begin{gathered}
B^{a b}=\frac{1}{\alpha^{2}+\beta^{2}}\left[-\frac{\alpha}{2} \epsilon^{a b c d} F_{c d}+\beta F^{a b}\right] \\
B^{a 4} \equiv B^{a}=\frac{1}{\beta} F^{a 4}=\frac{1}{\beta l} d_{\omega} e^{a}
\end{gathered}
$$

\section{A equations}

$$
\left(d_{\mathrm{A}} \mathrm{B}\right)^{I J}=\frac{1}{2} J_{P}^{I J}(x)
$$

where we have introduced the three-form

$$
J_{P}^{I J}(x)=\int \epsilon_{\mu \nu \rho \sigma} J^{I J}(\tau) \dot{z}^{\sigma} \delta^{4}(x-z(\tau)) d x^{\mu} \wedge d x^{\nu} \wedge d x^{\rho}
$$

with $\epsilon_{\mu \nu \rho \sigma}$ the Levi-Civita tensor $\epsilon_{0123}=1$. This form is such that

$$
\int J_{P}^{I J}(x) \wedge a(x)=\int_{P} d \tau J^{I J}(\tau) \dot{z}^{\mu}(\tau) a_{\mu}
$$

for any one form $a$.

Finally we have 
h equations, obtained by varying the action with respect to an $S O(4,1)$ transformation (2.15).

$$
\alpha \epsilon^{a b c d} B_{a b} \wedge B_{c}=\left(\int d \tau\left(D_{\tau} J\right)^{d} \delta^{4}(x-z(\tau))\right) d^{4} x
$$

Let us pause for a moment to recall that in the case of a particle in external, fixed gravitational field the $h$ equation of motion (3.26), for $\mathrm{h} \in \mathrm{SO}(4,1)$ has led to constraints imposed on components of gravitational field strengths. As we will see in a moment this problem is absent if the gravitational field is dynamical, in the case $\alpha \neq 0$. Indeed, consider eq. (4.3). Applying the covariant derivative to both sides we get

$$
\left(d_{\mathrm{A}} d_{\mathrm{A}} \mathrm{B}\right)^{I J}=\frac{1}{2}\left(d_{\mathrm{A}} \mathrm{J}_{P}\right)^{I J}(x)=-\frac{1}{2}\left(\int D_{\tau} \mathrm{J}(\tau) \delta^{4}(x-z(\tau)) d \tau\right)^{I J}
$$

Therefore the component $a 4$ of (4.7) is just $-1 / 2$ times the RHS of eq. (4.6). Now

$$
\left(d_{\mathrm{A}} d_{\mathrm{A}} \mathrm{B}\right)^{I J}=F_{K}^{I} \wedge B^{K J}+F^{J}{ }_{K} \wedge B^{I K}
$$

To compare this with eq. (4.6) we just need the translational component of this:

$$
\begin{gathered}
F_{c}^{d} \wedge B^{c 4}+F^{4}{ }_{c} \wedge B^{d c}=\left(B^{c d}-\frac{1}{\beta} F^{c d}\right) \wedge \frac{1}{l} d_{\omega} e_{c}= \\
=-\frac{\alpha}{\beta} \frac{1}{\alpha^{2}+\beta^{2}}\left(\alpha F^{c d}+\frac{\beta}{2} \epsilon^{c d a b} F_{a b}\right) \wedge \frac{1}{l} d_{\omega} e_{c} \\
=-\frac{\alpha}{2} \epsilon^{a b c d}\left(B_{a b} \wedge B_{c}\right) .
\end{gathered}
$$

This is just $-1 / 2$ times the LHS of eq. (4.6). Thus we conclude that the $h$ equation (4.6) is just a part of integrability conditions of (4.3). Thus in what follows we can disregard eq. (4.6) whatsoever.

We see therefore that in the case, in which gravity is fully dynamical, it is consistent to take the gauge degrees of freedom that become dynamical at the particle world-line, described by $\mathrm{h} \in \mathrm{SO}(4,1)$. Diffeomorphisms and those $\mathrm{h}$ that belong to $\mathrm{SO}(3,1)$ leave the bulk action invariant, and therefore they are dynamical degrees of freedom only along the worldline location. The analysis of their dynamics is not modified by the coupling to gravity and the results of the previous section therefore apply in the case where gravity is dynamical. Moreover, these dynamical degree of freedom ensures that the all formalism is invariant under the usual gauge group of gravity.

Those $\mathrm{h}$ that belong to $\mathrm{SO}(4,1) / \mathrm{SO}(3,1)$ do not leave the bulk action invariant, and therefore they are dynamical degrees of freedom even in the 
absence of the particle. Since their equation of motion is a subset of Einstein equation, this suggests that they are determined on-shell by the gravitational and particle degree of freedom. However, this determination which can be performed in the pure gravitational case [1] is more involved and not yet known when matter is present.

Let us now consider the equation for gravitational field produced by a point particle in full generality. Our starting point will be eq. (4.1), (4.2), (4.3), Consider first equation (4.3) in 'rotational' direction and expand its RHS.

$$
\begin{aligned}
\left(d_{\mathrm{A}} \mathrm{B}\right)^{a b} & =d_{\omega} B^{a b}+A^{a 4} \wedge B_{4}{ }^{b}+A^{b 4} \wedge B^{a}{ }_{4} \\
& =d_{\omega} B^{a b}+\frac{1}{\beta l^{2}}\left(T^{a} \wedge e^{b}-e^{a} \wedge T^{b}\right) .
\end{aligned}
$$

Now, from definition of $F$ (2.9)

$$
d_{\omega} F^{a b}=d_{\omega} R^{a b}-\frac{1}{l^{2}}\left(T^{a} \wedge e^{b}-T^{b} \wedge e^{a}\right)=-\frac{1}{l^{2}}\left(T^{a} \wedge e^{b}-T^{b} \wedge e^{a}\right)
$$

Thus

$$
\left(d_{\mathrm{A}} \mathrm{B}\right)^{a b}=d_{\omega}\left(B^{a b}-\frac{1}{\beta} F^{a b}\right)=-\frac{\alpha}{\beta} \frac{1}{\alpha^{2}+\beta^{2}} d_{\omega}\left(\alpha F^{a b}+\frac{\beta}{2} \epsilon^{a b c d} F_{c d}\right)=\frac{1}{2} J_{P}^{a b}
$$

and eq. (4.3) can be written as

$$
T^{a} \wedge e^{b}-T^{b} \wedge e^{a}=\frac{\beta l^{2}}{2 \alpha}\left(\alpha \delta_{c d}^{a b}-\frac{\beta}{2} \epsilon_{c d}^{a b}\right) J_{P}^{c d}
$$

This is an algebraic equation which fully determine the torsion in term of the spin of the particle. When written in terms of the gravitational parameter it reads

$$
T^{a} \wedge e^{b}-T^{b} \wedge e^{a}=\frac{G \gamma}{2\left(1+\gamma^{2}\right)}\left(\delta-\frac{\gamma}{2} \epsilon\right)^{a b}{ }_{c d} s_{P}^{c d}
$$

and we see that the Immirzi parameter affects the coupling between torsion and spin; in the case of usual metric gravity $\gamma=0$ the torsion is zero.

If we now consider the translation part of (4.3) we get

$$
\begin{aligned}
\left(d_{\mathrm{A}} \mathrm{B}\right)^{a 4} & =d_{\omega} B^{a 4}+A^{4}{ }_{b} \wedge B^{a b} \\
& =\frac{1}{l}\left(\frac{1}{\beta} d_{\omega} T^{a}-B^{a b} \wedge e_{b}\right) \\
& =\frac{1}{l}\left(\frac{1}{\beta} F^{a b}-B^{a b}\right) \wedge e_{b}
\end{aligned}
$$


Thus the field equation gives us the Einstein equation

$$
\frac{\alpha}{\left(\alpha^{2}+\beta^{2}\right)}\left(\frac{\alpha}{\beta} R^{a b} \wedge e_{b}+G^{a}\right)=\frac{l}{2} J_{P}^{a}
$$

where

$$
G^{a} \equiv \frac{1}{2} \epsilon^{a b c d} F_{c d} \wedge e_{b}=\frac{1}{2} \epsilon^{a b c d}\left(R_{c d} \wedge e_{b}-\frac{\Lambda}{3} e_{b} \wedge e_{c} \wedge e_{d}\right)
$$

is the Einstein tensor with cosmological constant. The first term on the RHS is the derivative of the torsion $d_{\omega}^{2} e^{a}=d_{\omega} T^{a}=R^{a b} \wedge e_{b}$. Equation (4.14) written in terms of the gravity coupling constant (2.12) reads

$$
\frac{1}{\gamma} R^{a b} \wedge e_{b}+G^{a}=\frac{G}{2} p_{P}^{a}
$$

Equations (4.12), (4.15) characterize the gravitational field produced by point particle with momentum $p^{a}$ and spin $s^{a b}$, and have the standard form.

\section{Quantum Particles and Wilson lines}

Let us now describe the effect of quantizing particle degrees of freedom. We first restrict our analysis to case of the pure BF-theory $(\alpha=\beta=0)$ to show that in the quantum regime inclusion of the particle is realized exactly by insertion of Wilson lines. Indeed in this case the bulk action is invariant under $\mathrm{SO}(4,1)$ gauge transformation, while the insertion of the particle breaks this symmetry at the location of the particle. The $\mathrm{SO}(4,1)$ gauge group element $h(\tau)$ at the location of the particle becomes a dynamical object and its integration in the path integral gives

$$
W(A)=\int \mathcal{D}(\mathrm{h}) e^{i \int_{P}-\operatorname{Tr}\left(\mathrm{KA}_{\tau}^{\mathrm{h}}(\tau)\right)}
$$

with $A^{h}=h^{-1} A h+h^{-1} d h$. We can interpret this path integral as the quantum amplitude associated with the system described by the particle action

$$
S(\mathrm{~h})=\int \mathrm{d} \tau \operatorname{Tr}\left(\mathrm{h}^{-1} \dot{\mathrm{h} K}\right)+\operatorname{Tr}(\mathrm{AJ})
$$

where $J=\mathrm{h} K \mathrm{~h}^{-1}$.

The phase space variables of this system are $(h, J)$ satisfying the commutation relations (see [6])

$$
\left\{\mathrm{h}, J_{I J}\right\}=\gamma_{I J} \mathrm{~h}, \quad\left\{\mathrm{~J}_{I J}, \mathrm{~J}_{K L}\right\}=\eta_{J K} \mathrm{~J}_{I L}+\cdots
$$


and subject to the constraints

$$
C \equiv \mathrm{K}-\mathrm{h} J \mathrm{~h}^{-1}=0 .
$$

Among these constraints, 2 are first class $\left(C_{04}, C_{23}\right)$ and the others are second class. The algebra of gauge invariant observables commuting with the constraints is generated by $J_{I J}$ and their Dirac bracket is equal to the original bracket. They form an $\mathrm{SO}(4,1)$ algebra subject to the first class constraints (3.9), (3.10) which label a representation of mass $m$ and spin $s$. Due to the Feynman-Kac correspondence the path integral (5.1) computes, for a closed loop, the trace in the so $(4,1)$ representation $(m, s)$ of the Wilson line operator

$$
W(A)=\operatorname{Tr}_{(m, s)}\left(P \exp \int_{P} A_{I J} J^{I J}\right) .
$$

This correspondence between path integral and Wilson lines has been studied in the compact group case by Alekseev et al [10].

Let us remark that this gives an interesting perspective on Feynman amplitudes: Such amplitudes are related to path integrals (5.1) along the Feynman graph. The Feynman graph amplitude can thus be interpreted as a spin network evaluation (which generalizes Wilson loops) of a $\mathrm{SO}(4,1)$ spin network whose edges are labeled by pairs $(m, s)$.

Now if we turn on gravity $\alpha \neq 0$ the quantization story is more involved. In this case the bulk action is invariant only under $\mathrm{SO}(3,1)$ gauge transformations and the 'Wilson line' is now deformed by the coupling to the gravitational field, it depends on the value of the $B$ fields and becomes

$$
W(A)=\int \mathcal{D}(\mathrm{h}) e^{i \int_{P}-\operatorname{Tr}\left(\mathrm{KA}_{\tau}^{\mathrm{h}}(\tau)\right)+\alpha \int_{M} \operatorname{Tr}(\gamma(\mathrm{h}) B \wedge B)}
$$

where $\gamma(\mathrm{h})=\mathrm{h} \gamma \mathrm{h}^{-1}$.

\section{Conclusions}

In this paper we investigated the coupling of point particle to gravity regarded as a constrained topological field theory. Our results presented here could be treated as a starting point for various directions of investigations.

First, since the $\alpha$ parameter is small, we can consider a perturbation theory of gravity coupled to particle(s) being the perturbation theory in $\alpha$. The distinguished feature of this theory would be that it is, contrary to earlier approaches, manifestly diffeomorphism-invariant, so its framework it is possible to talk about weak gravitational field in the conceptual framework 
of full general relativity. These investigations, both in the case of $\beta=0$ and $\beta \neq 0$ will be presented in the forthcoming paper.

The fuller control over the small $\alpha$ sector will presumably make it possible to address the outstanding question of what is the flat space limit of the theory of quantum gravity, coupled to point particles. It has been claimed that such a theory will be not the special relativity, but some form of doubly special relativity (see e.g. 11.). This has been shown in the case of three dimensional gravity [7. Indeed the key ingredient that allowed to construction the effective dynamic of matter coupled to $3 \mathrm{~d}$ quantum gravity was the understanding that particles can be described as charged topological defect. This allowed to quantize in one stroke gravity and matter and simplify drastically the problem of understanding the modification of matter dynamics due to quantum gravity effects. We hope that the results presented here will similarly simplify the study of quantum gravity to matter fields, especially in the limit where $\alpha$ is small, and according to the perturbation proposed in [1].

Last but not least there is a curious appearance of gravitational analogues of magnetic monopole in the topological sector of our theory, with $\alpha=0, \beta \neq 0$. Consider eq. (4.3)

$$
\left(d_{\mathrm{A}} \mathrm{B}\right)^{I J}=\frac{1}{2} J_{P}^{I J}(x)
$$

where $J_{P}^{I J}(x)$ is defined by (4.4). Since for $\alpha=0, B^{I J}=1 / \beta F^{I J}$, it seems for the first sight that eq. (6.1) is simply inconsistent because after substituting $\mathrm{F}$ for $\mathrm{B}$ the left hand side is identically zero by Bianchi identity. However having point-like, distributional sources we can allow for distributional connections, for which Bianchi identity does not hold, as in the case of Dirac monopole.

By construction (cf. eq. 3.5) there is always a gauge transformation which makes it possible to fix the gauge such that

$$
\frac{1}{\beta}\left(d_{\mathrm{A}} \mathrm{F}\right)^{I J}=\frac{1}{2} K^{I J} \delta_{P}
$$

where $\mathrm{K}$ is given by eq. (3.2). It is now clear that a solution of (6.1) has the form

$$
\mathrm{A}=\frac{\beta}{2} \mathrm{~K} A_{D}
$$

where $A_{D}$ is the abelian Dirac monopole connection (see for example [12]). For example in the patch which does not cover the negative $z$ axis

$$
A_{D}=\frac{x d y-y d x}{r(r+z)}
$$


in cartesian coordinates.

To construct a general solution let us assume that

$$
\mathrm{A}=\mathrm{A}_{0}+\frac{\beta}{2} \mathrm{~K} A_{D} \equiv \mathrm{A}_{0}+\frac{\beta}{2} \mathrm{~A}_{D}
$$

where $A_{0}$ is an arbitrary non-singular connection (i.e., the one that satisfies Bianchi identity $d_{A_{0}} F\left(A_{0}\right)=0$.) Plugging this anzatz into eq. (6.2) we find that this equation is satisfied identically.

What is even more interesting, the monopole configurations arising in the topological limit of gravity give rise to correct particle action. To see this, consider the action (2.4) in the $\alpha=0$ case

$$
S=\int\left(B_{I J} \wedge F^{I J}-\frac{\beta}{2} B^{I J} \wedge B_{I J}\right)
$$

Solving for $\mathrm{B}$, and plugging the solution back to the action, we find

$$
S=\frac{1}{2 \beta} \int\left(F_{I J} \wedge F^{I J}\right)
$$

Using (6.1), integrating the delta, and going to arbitrary gauge we get

$$
S=\frac{1}{4} \int \operatorname{Tr}\left(\mathrm{A}^{\mathrm{h}}(\tau) K\right)+C S(\mathrm{~A})
$$

where $C S(\mathrm{~A})$ is the boundary Chern-Simon action on $S^{2} \times R$ (spacelike infinity times time.) We see therefore that the particle action arises from singularities of topological remnant of gravitational field, of the form of generalized monopoles. It is not clear however, if this type of construction can be extended to the full theory, with $\alpha \neq 0$.

\section{Acknowledgements}

For JK-G this work is partially supported by the grants KBN 1 P03B 01828 and University of Wroclaw 2594/W/IFT.

\section{Appendix}

In this appendix, we recall our conventions and present some useful formulas.

For gamma-matrices, we use

$$
\left\{\gamma^{a}, \gamma^{b}\right\}=2 \eta^{a b}
$$


where $\eta^{a b}$ is the Minkowski metric of signature $(-,+,+,+)$. For commutator of two gamma matrices, we use the notation

$$
\gamma^{a b}=\frac{1}{2}\left[\gamma^{a}, \gamma^{b}\right]
$$

We denote "gamma-five" matrix by $\gamma$ :

$$
\gamma=-i \gamma^{0} \gamma^{1} \gamma^{2} \gamma^{3}, \quad\left\{\gamma, \gamma^{a}\right\}=\left\{\gamma, \gamma^{a b}\right\}=0,
$$

which satisfies $\gamma^{2}=1$. The matrices

$$
\gamma^{a_{1} \ldots a_{n}}=\frac{1}{n !}\left(\gamma^{a_{1}} \cdots \gamma^{a_{n}} \pm \text { permutations }\right)
$$

satisfy the identities

$$
\begin{gathered}
\gamma^{a} \gamma^{b}=\gamma^{a b}+\eta^{a b} \\
\gamma^{a b} \gamma^{c}=\gamma^{a b c}+\eta^{b c} \gamma^{a}-\eta^{a c} \gamma^{b} \\
\gamma^{a b c} \gamma^{d}=\epsilon^{a b c d} \gamma+\eta^{d c} \gamma^{a b}-\eta^{b d} \gamma^{a c}-\eta^{a d} \gamma^{c b} \\
\gamma_{a b} \gamma=\frac{i}{2} \epsilon_{a b c d} \gamma^{c} \gamma^{d}, \gamma_{a b c}=i \epsilon_{a b c d} \gamma^{d} \gamma \\
\left\{\gamma_{a} \gamma, \gamma\right\}=0,\left\{\gamma_{a b}, \gamma\right\}=i \epsilon_{a b c d} \gamma^{c d} \\
\left\{\gamma_{b} \gamma, \gamma_{a}\right\}=i \epsilon_{a b c d} \gamma^{c d},\left\{\gamma_{b c}, \gamma_{a}\right\}=2 i \epsilon_{a b c d} \gamma^{d} \gamma
\end{gathered}
$$

$\gamma^{a} \gamma_{a}=4, \quad \gamma^{a_{1} \ldots a_{n} b} \gamma_{b}=(4-n) \gamma^{a_{1} \ldots a_{n}} \quad \gamma^{b} \gamma^{a_{1} \ldots a_{n}} \gamma_{b}=(-1)^{n}(4-2 n) \gamma^{a_{1} \ldots a_{n}}$

The Lie algebra so $(4,1)$ is generated by $T_{a b}=\gamma_{a b} / 4$ and $T_{a}=\gamma_{a} \gamma / 4$ which satisfy

$$
\begin{aligned}
2\left[T_{a b}, T_{c d}\right] & =\eta_{b c} T_{a d}-\eta_{a c} T_{b d}-\eta_{b d} T_{a c}+\eta_{a d} T_{b c} \\
2\left[T_{a b}, T_{c}\right] & =\eta_{b c} T_{a}-\eta_{a c} T_{b} \\
2\left[T_{a}, T_{b}\right] & =-T_{a b} .
\end{aligned}
$$

Moreover

$$
\operatorname{Tr}\left(T_{a b} T_{c d}\right)=\frac{1}{4}\left(\eta_{b c} \eta_{a d}-\eta_{a c} \eta_{b d}\right), \quad \operatorname{Tr}\left(T_{a} T_{b}\right)=-\frac{1}{4} \eta_{a b} .
$$

The normalisation of the generators is such that if $\mathrm{A}=A^{I J} T_{I J}, \mathrm{~B}=B^{I J} T_{I J}$ then

$$
[\mathrm{A}, \mathrm{B}]=\left(A^{I K} B_{K}^{J}-B^{I K} A_{K}^{J}\right) T_{I J}=[\mathrm{A}, \mathrm{B}]^{I J} T_{I J}
$$




\section{References}

[1] L. Freidel and A. Starodubtsev, "Quantum gravity in terms of topological observables," arXiv:hep-th/0501191.

[2] A.P. Balachandran, G. Marmo, B.-S. Skagerstam, and A. Stern Gauge Symmetries and Fibre Bundles. Application to Particle Dynamics, Lecture Notes in Physics 188, Springer, 1983, and references therein.

[3] S.W. MacDowell, F. Mansouri, "Unified Geometric theory of gravity and supergravity". Phys. Rev. Lett. 38 739, 1977., Erratum-ibid. 38 1376, 1977.

[4] A. Perez and C. Rovelli, "Physical effects of the Immirzi parameter," arXiv:gr-qc/0505081, L. Freidel, D. Minic and T. Takeuchi, "Quantum gravity, torsion, parity violation and all that," Phys. Rev. D 72, 104002 (2005) arXiv:hep-th/0507253. A. Randono, "A note on parity violation and the Immirzi parameter," arXiv:hep-th/0510001.

[5] L. Smolin and A. Starodubtsev, "General relativity with a topological phase: An action principle," arXiv:hep-th/0311163.

[6] P. de Sousa Gerbert, "On Spin And (Quantum) Gravity In (2+1)Dimensions," Nucl. Phys. B 346, 440 (1990).

[7] L. Freidel and E. R. Livine, "Ponzano-Regge model revisited. III: Feynman diagrams and effective field theory," Class. Quant. Grav. 23 (2006) 2021 arXiv:hep-th/0502106 . L. Freidel and E. R. Livine, "Effective 3d quantum gravity and non-commutative quantum field theory," arXiv:hep-th/0512113.

[8] J. C. Baez and A. Perez, "Quantization of strings and branes coupled to BF theory," arXiv:gr-qc/0605087.

[9] M. Mathisson, Acta. Phys. Polon. 6 (1937) 225; A. Papapetrou, "Spinning Test Particles In General Relativity. 1," Proc. Roy. Soc. Lond. A 209 (1951) 248.

[10] A. Alekseev, L. D. Faddeev and S. L. Shatashvili, "Quantization Of Symplectic Orbits Of Compact Lie Groups By Means Of The Functional Integral," J. Geom. Phys. 5 (1988) 391.

[11] J. Kowalski-Glikman, "Doubly special relativity: Facts and prospects," arXiv:gr-qc/0603022. 
[12] M. Nakahara, Geometry, Topology, and Physics, II ed., IoP Publishing, Bristol, 2005. 